\title{
Weiterempfehlungsprogramme: Die Wirkung des Akquisitionskanals und der Prämienaufteilung auf den Erfolg von Weiterempfehlungen
}

\author{
Lisa-Marie Klopfer • Martina Steul-Fischer
}

Online publiziert: 25. Juni 2020

(C) Der/die Autor(en) 2020, korrigierte Publikation 2021

Zusammenfassung Weiterempfehlungsprogramme haben sich in der Versicherungsbranche als Marketinginstrument etabliert, da sie sich als effektive und kosteneffiziente Alternative zu herkömmlichen Maßnahmen der Kundenakquise und -bindung, wie beispielsweise Direktmarketing, erweisen. Der Fokus der bisherigen Forschung zu Weiterempfehlungen und Weiterempfehlungsprogrammen lag primär auf der Weiterempfehlungsbereitschaft des Bestandskunden. Einer erfolgreichen Weiterempfehlung bedarf es jedoch auch der Bereitschaft zur Empfehlungsannahme des Empfehlungsempfängers. Diese empirische Studie rückt daher den Empfehlungsempfänger und die Determinanten der Annahme einer Weiterempfehlung in den Vordergrund. Die Ergebnisse zeigen, dass Kunden, die selbst über das Weiterempfehlungsprogramm eines Unternehmens akquiriert wurden, im Vergleich zu anderweitig gewonnenen Kunden (z. B. Direktmarketing) eine höhere Erfolgswahrscheinlichkeit generieren (Akquisitionskanal). Weiterhin führt das Teilen der Weiterempfehlungsprämie zu einer höheren Erfolgswahrscheinlichkeit als das alleinige Entlohnen des Empfehlungsgebers (Prämienaufteilung). Dieser Wirkungszusammenhang wird durch die wahrgenommene Fairness der Prämienaufteilung mediiert.

Lisa-Marie Klopfer $(\varangle) \cdot$ Martina Steul-Fischer Lehrstuhl für BWL, insb. Versicherungsmarketing, Fachbereich Wirtschaftsund Sozialwissenschaften, Friedrich-Alexander-Universität Erlangen-Nürnberg, Lange Gasse 20, 90403 Nürnberg, Deutschland E-Mail: lisa-marie.klopfer@fau.de 


\title{
Customer referral programs: the effects of acquisition channel and reward allocation on referral acceptance
}

\begin{abstract}
Referral programs are established as a marketing tool in the insurance industry, as they offer an effective and cost-efficient alternative to traditional methods of customer acquisition and retention, such as direct marketing. Previous research on referrals and referral reward programs primarily focused on the referral likelihood of existing customers. However, successful referrals also require the willingness of the recipient to accept the referral. This empirical study therefore focuses on the referral recipient and the determinants of referral acceptance. The results reveal that customers who have been acquired through a company's referral reward program (acquisition channel) generate a higher likelihood of successful referral compared to customers acquired through other marketing activities (e.g. direct marketing). Furthermore, sharing the referral reward between sender and receiver of the referral leads to a higher likelihood of successful referral than rewarding the referrer only (reward allocation). This effect is mediated by the perceived fairness of the reward allocation.
\end{abstract}

\section{Einführung}

Einen Konsumenten erreichen täglich im Durchschnitt 10.000 unterschiedliche Werbebotschaften (Koch 2018). Dies führt, gepaart mit der stetig steigenden Produktvielfalt, zu einer Informationsüberflutung und letztendlich zu einer Überforderung potenzieller Kunden (Barrot et al. 2013). Konsumenten beziehen daher die Meinungen und die Erfahrungen ihrer Mitmenschen mit in die Kaufentscheidung ein und vertrauen Weiterempfehlungen als interpersonale Informationsquelle häufig mehr als Marketingversprechen (Keller 2007). In der Versicherungsbranche, wie ebenso in anderen Dienstleistungssektoren, wird Weiterempfehlungen eine besondere Bedeutung zugeschrieben, da Dienstleistungen aufgrund ihrer Nichtgreifbarkeit ein erhöhtes subjektiv wahrgenommenes Kaufrisiko aufweisen und Entscheidungsunsicherheiten hervorrufen (Murray 1991; Zeithaml 1981). Zur Vorbeugung potenzieller Fehlentscheidungen und Verluste werden von den potenziellen Neukunden daher direkte oder indirekte Fremderfahrungen mit dem Dienstleistungsanbieter herangezogen (Laroche et al. 2004; Murray 1991; Wirtz et al. 2013).

Durch die Implementierung sogenannter Weiterempfehlungsprogramme sollen die Bestandskunden zur Abgabe von Weiterempfehlungen angeregt werden (Ryu und Feick 2007). Als Hybrid zwischen dem traditionellen Marketing und der natürlich auftretenden interpersonalen Kommunikation werden die Programme wie andere Marketingaktivitäten unternehmensseitig initiiert, jedoch verläuft die Durchführung dieser Marketingmaßnahme über die sozialen Verbindungen der Bestandskunden (Godes und Mayzlin 2009). Digitale Technologien und das Internet haben zu einer Verlagerung der Weiterempfehlungsprogramme in die Online-Welt geführt und deren Verbreitung beschleunigt (Wirtz et al. 2013). Die Teilnahme an den Programmen erfolgt zunehmend via E-Mail oder per Online-Formular auf der Unternehmensweb- 
site, aber auch vermehrt durch das Versenden von Links in sozialen Netzwerken wie WhatsApp oder Facebook (Barrot et al. 2013).

In der Versicherungsbranche haben sich Weiterempfehlungsprogramme bereits als vielversprechendes Marketinginstrument etabliert, da sie sich als effektive und kosteneffiziente Alternative zu herkömmlichen Maßnahmen der Neukundenakquise, wie beispielsweise Direktmarketing, erweisen (Garnefeld et al. 2011; Xiao et al. 2011). Innerhalb sozialer Beziehungen ist davon auszugehen, dass sich Individuen im Hinblick auf Bedürfnisse sowie Interessen ähnlich sind und der Empfehlungsgeber deshalb die Bedürfnisstruktur des Empfehlungsempfängers kennt (Armelini et al. 2015; Schmitt et al. 2011). Der Einbezug dieses Wissens in die Weiterempfehlung und die erhöhte Glaubwürdigkeit persönlicher Empfehlungen begründen die Effektivität der Weiterempfehlungsprogramme (Allsop et al. 2007; Garnefeld et al. 2013; Xiao et al. 2011). Da der Prozess der Empfehlung in dem sozialen Netzwerk des Empfehlungsgebers abläuft, hat ein Unternehmen lediglich die Kosten der Implementierung des Programms, der Verwaltung, der Bestandsführung sowie der laufenden Prämienauszahlungen zu tragen (Garnefeld et al. 2011; Schmitt et al. 2011). Schmitt et al. (2011) haben gezeigt, dass die Akquisitionskosten für empfohlene Neukunden im Vergleich zu Neukunden, die über andere Marketingmaßnahmen akquiriert wurden, durchschnittlich um ca. $20 €$ geringer sind. Zudem bedingt die Auszahlung der Weiterempfehlungsprämie im Normalfall gleichzeitig eine Umsatzgenerierung durch den neu geworbenen Kunden (Biyalogorsky et al. 2001). Zusammengefasst spricht dies für die Kosteneffizienz der Weiterempfehlungsprogramme.

Existierende Studien zeigen, dass empfohlene Neukunden kurz- und langfristig einen höheren Kundenwert generieren, einen höheren Deckungsbeitrag sowie ein geringeres Kündigungsrisiko aufweisen, als Neukunden, die über traditionelle Marketingkanäle akquiriert werden (Armelini et al. 2015; Schmitt et al. 2011; Villanueva et al. 2008). Neben dem Einsatz zur Kundenakquise sind Weiterempfehlungsprogramme aufgrund der gesteigerten Loyalität von Neukunden sowie Bestandskunden ebenfalls ein wichtiges Instrument der Kundenbindung (Garnefeld et al. 2013; Ryu und Feick 2007).

Das Ziel der vorliegenden Studie ist es, den Einfluss des Akquisitionskanals des Empfehlungsgebers (Weiterempfehlungsprogramm vs. Direktmarketing) und der Prämienaufteilung (Prämie für den Empfehlungsgeber vs. Teilen zwischen Empfehlungsgeber und -empfänger) auf die Erfolgswahrscheinlichkeit einer Weiterempfehlung zu analysieren. Im zweiten Kapitel werden zunächst die theoretischen und konzeptionellen Grundlagen erläutert. Anschließend wird im Rahmen einer empirischen Studie gezeigt, inwieweit die Erfolgswahrscheinlichkeit einer Weiterempfehlung von den genannten Determinanten beeinflusst wird. Des Weiteren wird untersucht, ob der Einfluss der Prämienaufteilung auf die Wahrscheinlichkeit einer erfolgreichen Weiterempfehlung durch die wahrgenommene Fairness der Prämienaufteilung mediiert wird. Aus der Diskussion der Ergebnisse erfolgen Implikationen für Forschung und Praxis. Abschließend werden die Limitationen dieser Studie und der zukünftige Forschungsbedarf aufgezeigt. 


\section{Theoretische und konzeptionelle Grundlagen}

Weiterempfehlungsprogramme als eine Art der stimulierten Weiterempfehlung zeichnen sich durch drei charakteristische Eigenschaften aus (Schmitt et al. 2011). Zum einen sind die Programme von Unternehmen bewusst initiiert, werden aktiv vorangetrieben und können kontinuierlich überprüft und optimiert werden (Schmitt et al. 2011). Bei organischen Weiterempfehlungen, wie beispielsweise in spontanen Gesprächen, sind vor allem die Kontrolle und Verwaltung der Empfehlungen nicht gegeben, da dem Unternehmen keinerlei individuelle Daten zu bestehenden Kundenbeziehungen vorliegen (Schmitt et al. 2011). Innerhalb der Weiterempfehlungsprogramme kann im Vergleich dazu sichergestellt werden, dass ein weiterempfohlener Kunde nicht bereits Kunde des Anbieters ist bzw. war. Das zweite wichtige Merkmal der Programme stellt die Nutzung der sozialen Verbindungen der Bestandskunden zur Gewinnung potenzieller Neukunden dar (Garnefeld et al. 2013). Um eine positive Weiterempfehlung zu stimulieren, bieten die Unternehmen ihren Kunden drittens extrinsische Motivationsanreize, die erfolgreiche Produktkäufe bzw. Vertragsabschlüsse und somit die Neukundenakquise belohnen (Schmitt et al. 2011; Wirtz und Chew 2002). Weiterempfehlungsprämien können sowohl monetärer (z.B. Geldprämie) als auch nicht-monetärer (z.B. Sachprämien, Spenden) Natur sein (Jin und Huang 2014; Verlegh et al. 2013). Im Regelfall wird lediglich der Empfehlungsgeber (d.h. der Bestandskunde) honoriert, einige Unternehmen belohnen hingegen beide Parteien (d.h. Empfehlungsgeber und Empfehlungsempfänger) für die Teilnahme an dem Weiterempfehlungsprogramm (Verlegh et al. 2013). Da die Ausgestaltung der Programme den Unternehmen selbst unterliegt, gibt es zudem Unterschiede hinsichtlich der Art der Weiterempfehlung, die dem Bestandskunden eine Weiterempfehlungsprämie sichert. Während im Normalfall erst der Vertragsabschluss eine erfolgreiche Empfehlung darstellt, belohnen andere Firmen bereits das Senden von Weiterempfehlungen per E-Mail oder das Bereitstellen von Kontaktdaten potenziell interessierter Familienmitglieder, Freunde oder Bekannte (Wirtz und Chew 2002).

Die bisherige Forschung hat sich primär mit den Determinanten der Abgabe einer Weiterempfehlung befasst. Die Weiterempfehlungsbereitschaft des Empfehlungsgebers wird unter anderem durch die Beziehungsstärke zu dem Empfehlungsempfänger (u. a. Brown und Reingen 1987), die Kundenzufriedenheit (u. a. Wirtz und Chew 2002) sowie durch die Anwesenheit, Höhe und Aufteilung der Weiterempfehlungsprämie (u. a. Ryu und Feick 2007) beeinflusst. Einem erfolgreichen und effektiven Weiterempfehlungsprogramm bedarf es jedoch, neben der Weiterempfehlungsbereitschaft des Bestandskunden, vor allem der Bereitschaft zur Empfehlungsannahme auf Seiten des Empfehlungsempfängers (Verlegh et al. 2013). Diese Studie rückt daher den Empfehlungsempfänger und die Determinanten des Erfolgs einer Weiterempfehlung, d.h. Wechsel des Dienstleistungsanbieters in Folge der Empfehlung, in den Vordergrund. Im Folgenden werden die identifizierten Einflussfaktoren näher erläutert. 


\subsection{Der Akquisitionskanal des Empfehlungsgebers}

Obwohl viele Studien zu Weiterempfehlungsprogrammen den Empfehlungsgeber in den Fokus der Betrachtung rücken, wird dieser jedoch zumeist stark vereinfacht als Bestandskunde beschrieben und nicht weiter definiert. Lediglich von Wangenheim und Bayón (2004) teilen den von ihnen untersuchten Kundendatensatz in verschiedene Gruppen ein und betrachten deren Weiterempfehlungsverhalten differenziert. Grundsätzlich unterteilen sie den Kundenbestand in kürzlich zum Unternehmen gewechselte Neukunden und Bestandskunden, die noch keinen Anbieterwechsel vollzogen haben bzw. dieser bereits vor sehr langer Zeit stattfand (von Wangenheim und Bayón 2004). Eine weitere Unterscheidung erfolgt im Rahmen der Neukunden zwischen Kunden, die selbst über das Weiterempfehlungsprogramm des Unternehmens akquiriert wurden und Kunden, die über andere Vertriebskanäle gewonnen wurden (von Wangenheim und Bayón 2004). Die Ergebnisse der Untersuchung verdeutlichen die Wichtigkeit einer detaillierten Betrachtungsweise des Akquisitionskanals des Empfehlungsgebers. Neukunden, die aus dem Weiterempfehlungsprogramm des Unternehmens hervorgegangen sind, zeichnen sich im Vergleich zu den anderen Neukunden insbesondere durch eine höhere Zufriedenheit und Loyalität sowie eine erhöhte Anzahl positiver Weiterempfehlungen aus (von Wangenheim und Bayón 2004).

Diese Ergebnisse können hauptsächlich durch zwei Konstrukte begründet werden. Zum einen zeigen wissenschaftliche Studien, dass das Weiterempfehlungsverhalten von Konsumenten in der Zeit nach einem Produktkauf am höchsten ist (East et al. 2001; Richins und Bloch 1986). Durch den Kauf eines Gutes oder den Wechsel des Anbieters befinden sich Neukunden in einem Stadium des erhöhten situativen Involvements, welches aus der aktiven Kaufentscheidung, der Neuheit des Produktes oder aufgrund kognitiver Dissonanz resultieren kann (Homburg 2017; Richins und Bloch 1986). Von Wangenheim und Bayón (2007) haben die Verbindung zwischen einer kürzeren Beziehung zum Unternehmen, dem gesteigerten situativen Involvement und der demzufolge erhöhten Anzahl an Weiterempfehlungen nachgewiesen. Zum anderen weisen Neukunden, die selbst über ein Weiterempfehlungsprogramm gewonnen wurden, eine erhöhte Weiterempfehlungsbereitschaft im Verglich zu anderweitig akquirierten Neukunden auf (Villanueva et al. 2008). Begründet liegt dies in der Erfahrung der eigenen Teilnahme an dem Weiterempfehlungsprogramm, die sowohl zu einem besseren Verständnis für den Entscheidungs- und Teilnahmeprozess des Empfehlungsempfängers als auch zu einer Reduktion des wahrgenommenen Risikos einer Weiterempfehlung führt (Viswanathan et al. 2018; von Wangenheim und Bayón 2004). Nitzan und Libai (2011) haben zudem gezeigt, dass der Anbieterwechsel einer Person im näheren Umfeld eines Konsumenten mit einer hohen Wahrscheinlichkeit auch zu einem Wechsel dieses Konsumenten führen kann, da Individuen innerhalb eines sozialen Netzwerks dazu tendieren, sich unter anderem hinsichtlich der Einstellungen und Bedürfnisse zu ähneln. Basierend auf den wissenschaftlichen Erkenntnissen wird deshalb angenommen, dass der aktuelle Anbieterwechsel des Empfehlungsgebers auf eine positive, unterstützende Weise zu einem Wechsel des Empfängers der Weiterempfehlung beiträgt: 
$\mathbf{H}_{1}$ Neukunden, die über das Weiterempfehlungsprogramm des Unternehmens geworben wurden, generieren eine höhere Erfolgswahrscheinlichkeit einer Weiterempfehlung im Vergleich zu Neukunden, die über herkömmliche Akquisitionskanäle des Unternehmens geworben wurden.

\subsection{Die Prämienaufteilung zwischen Empfehlungsgeber und -empfänger}

Die Weiterempfehlungsprämie stellt einen wesentlichen Unterschied zwischen einem Weiterempfehlungsprogramm und der organischen Weiterempfehlung dar. Während das Aussprechen einer direkten Empfehlung lediglich zu einer Belohnung des Empfehlungsgebers auf psychologischer Ebene führen kann (Dichter 1966), ist die Weiterempfehlungsprämie, die in ihrer Art (monetär vs. nicht-monetär), Aufteilung (Empfehlungsgeber, Empfehlungsempfänger oder Beide erhalten eine Prämie) und Höhe vom Unternehmen festgelegt und variiert werden kann, ein attraktiver Anreiz zur Teilnahme an einem Weiterempfehlungsprogramm (Schmitt et al. 2011).

Aus verhaltensökonomischer Perspektive verändert die Anwesenheit einer Prämie die zugrundeliegende Interaktion zwischen Empfehlungsgeber und -empfänger; weg von dem sozialen Austauschprozess der natürlich auftretenden Weiterempfehlungen und hin zu einem marktähnlichen Verhalten mit Fokus auf Profitmaximierung (Heyman und Ariely 2004). Dies führt letztendlich dazu, dass sowohl der Empfehlungsgeber als auch der Empfehlungsempfänger extrinsische und intrinsische Motive in die Entscheidung hinsichtlich der Abgabe bzw. Annahme einer Weiterempfehlung einbeziehen (u.a. Kornish und Li 2010; Ryu und Feick 2007; Verlegh et al. 2013; Wirtz et al. 2013). Während der Empfehlungsgeber sich unter anderem um mögliche soziale Kosten der Weiterempfehlung und die eigene Reputation sorgt (Jin und Huang 2014; Xiao et al. 2011), versuchen Empfänger einer Empfehlung die (versteckten) Motive des Empfehlungsgebers einzuschätzen (Verlegh et al. 2013). Nachdem der Empfehlungsempfänger jedoch in den meisten Fällen nicht in der Lage ist, die Motive vollends vorherzusagen, kommt es häufig zu einem Gefühl der Unsicherheit (Sniezek und Van Swol 2001). Die darauffolgende Reaktanz in Bezug auf die Weiterempfehlung kann im schlimmsten Fall deren Ablehnung zur Folge haben (Sciandra 2019).

Wissenschaftliche Studien stellen zudem einen entscheidenden Zusammenhang zwischen der Beziehungsstärke sowie der vom Unternehmen festzulegenden Aufteilung der Prämie fest und zeigen deren gemeinsamen Einfluss auf die Weiterempfehlungs- bzw. Annahmewahrscheinlichkeit (u. a. Hong et al. 2017; Ryu und Feick 2007; Sciandra 2019; Verlegh et al. 2013). Weiterempfehlungen innerhalb starker Beziehungen finden vor allem aufgrund der Sorge um das Wohlergehen des Gegenübers statt, weshalb die Prämie geteilt oder dem Empfehlungsempfänger zugesprochen werden sollte (Ryu und Feick 2007; Verlegh et al. 2013). Weniger empfehlenswert ist es wiederum, den Empfehlungsgeber zu belohnen, da die Prämie nicht die Absicht der Weiterempfehlung darstellt (Ryu und Feick 2007). Hinsichtlich schwacher Beziehungen kann davon ausgegangen werden, dass ein bestehender Kunde ein Produkt oder Unternehmen nur weiterempfiehlt, wenn die Empfehlung dem Empfehlungsgeber selbst einen Vorteil einbringt (z.B. Prämie oder positiver Eindruck) (Verlegh et al. 2013; Xiao et al. 2011). Da ein Unternehmen jedoch lediglich die 
Wirkung einer Prämie beeinflussen kann, nicht aber die Reputation der Kunden, ist es sinnvoll, die Prämie entweder dem Empfehlungsgeber oder beiden Teilnehmern zuzusprechen, um die Wahrscheinlichkeit einer Weiterempfehlung zu erhöhen (Ryu und Feick 2007; Verlegh et al. 2013). Für die Teilung einer Prämie bei schwachen Beziehungen spricht das damit verbundene Aufrechterhalten der Glaubwürdigkeit einer Weiterempfehlung und deren Empfehlungsgeber (Kornish und Li 2010). Im Folgenden wird angenommen, dass die Weiterempfehlung innerhalb einer starken Beziehung (Strong Ties) stattfindet, da natürliche Weiterempfehlungen häufiger in starken Beziehungen auftreten (Ryu und Feick 2007). Strong Ties werden als Quelle für Empfehlungen bevorzugt und Empfehlungen bei dieser Beziehungsstärke werden im Allgemeinen als glaubwürdiger sowie einflussreicher eingestuft (Brown und Reingen 1987; Godes und Mayzlin 2009).

Da eine reine Belohnung des Empfehlungsempfängers in der Praxis selten angeboten wird (Ryu und Feick 2007), wird in der vorliegenden Studie lediglich zwischen der Prämie für den Empfehlungsgeber und dem Teilen der Weiterempfehlungsprämie unterschieden. Es wird erwartet, dass eine Weiterempfehlung mit einer höheren Wahrscheinlichkeit angenommen wird, wenn Empfehlungsgeber und -empfänger die Prämie teilen:

$\mathbf{H}_{2}$ Die Erfolgswahrscheinlichkeit einer Weiterempfehlung ist bei einer Prämienteilung höher im Vergleich zu keiner Prämienteilung.

\subsection{Die Rolle der wahrgenommenen Fairness im Hinblick auf die Prämienaufteilung}

Mit Blick auf die Aufteilung der Prämie ist die Thematisierung der wahrgenommenen Fairness unabdingbar. Die im Bereich des Konsumentenverhaltens viel erforschte Preisfairness bildet den Ausgangspunkt für die Annahme, dass die Fairness eine Determinante der Erfolgswahrscheinlichkeit einer Weiterempfehlung darstellt. Unter dem Begriff der Preisfairness versteht man im Allgemeinen die ,bewusst oder unbewusst von Gerechtigkeitsüberlegungen geprägten Wahrnehmungen der Transaktionsbedingungen und -abläufe“ (Diller 2002, S. 165). Da der Preis eines Produktes lediglich eine unbestimmte Information darstellt und somit für ein Fairnessurteil nicht ausreicht, vergleichen Konsumenten den Preis daher mit einem Referenzwert (Diller 2008). Im Regelfall ziehen Kunden in der Vergangenheit gezahlte Preise, Preise der Wettbewerber, von anderen Kunden bezahlte Preise oder den persönlichen ökonomischen und/oder sozialen Nutzen als Referenzpreis heran (Diller 2008; Maxwell et al. 1999). Da diese Preise von Konsument zu Konsument variieren, ist die wahrgenommene Preisfairness ein subjektives Konstrukt und von Person zu Person verschieden (Xia et al. 2004). Wird ein Preis schließlich als unfair wahrgenommen, hat dies negative Folgen für ein Unternehmen, wie beispielsweise eine Reduktion der Kaufintention, Beschwerden oder die Verbreitung von negativem Word-ofMouth (Xia et al. 2004).

Im vorliegenden Fall dient die Equity Theorie als Ansatz zur Begründung der erwarteten Fairnesswahrnehmungen der Empfehlungsempfänger, da sie die Relevanz der Gleichheit zwischen den Austauschpartnern betont (Diller 2008). Das Prinzip 
der Fairness in Anlehnung an die Equity Theorie sieht vor, dass sich die erbrachten Aufwendungen (Input) und die erhaltenen Erträge (Output) innerhalb einer sozialen Beziehung ausgleichen (Hong et al. 2017). Übertragen auf ein Weiterempfehlungsprogramm stellen die sozialen Kosten und die aufgewendete Zeit sowie die Mühe der Weiterempfehlung den Input der empfehlenden Person dar (Jin und Huang 2014; Ryu und Feick 2007). Die Prämie, die im Regelfall dem Empfehlungsgeber ausgezahlt wird, und eine potenzielle Steigerung der eigenen Reputation stellen den Output dar (Ryu und Feick 2007; Xiao et al. 2011). Für den Empfehlungsempfänger ist das dem Empfehlungsgeber entgegengebrachte Vertrauen in die Weiterempfehlung der Input und der Kauf eines neuen Produktes sowie gegebenenfalls (je nach Ausgestaltung des Weiterempfehlungsprogramms) eine Prämie der Output. Es wird deutlich, dass die Prämie einen wichtigen, wenn nicht sogar den wichtigsten Teil zu der wahrgenommenen Fairness eines Weiterempfehlungsprogramms beiträgt.

Betrachtet man nun lediglich die Prämienaufteilung im Kontext der Fairness, so wird eine gleichmäßige Teilung der Weiterempfehlungsprämie (50/50) zwischen Empfehlungsgeber und -empfänger als besonders fair angesehen (Hong et al. 2017). Jegliche Abweichung davon kann von dem Empfehlungsempfänger als unfair interpretiert werden und zu Abwehrreaktionen, wie das Ablehnen bzw. Nicht-Annehmen der Weiterempfehlung, führen (Sciandra 2019). Als wahrgenommener Preis dient dem Empfehlungsempfänger zumeist die Information über die Höhe und die Aufteilung der Prämie, die unter anderem der Weiterempfehlungsnachricht (z.B. via E-Mail) oder der Website des Unternehmens (z. B. Landingpage des Weiterempfehlungsprogramms) entnommen werden kann bzw. von dem Empfehlungsgeber preisgegeben wird. Den Referenzpreis sollte in der Regel der erwartete individuelle Nutzen des Empfehlungsempfängers darstellen, der im Falle eines Weiterempfehlungsprogramms dem Wechsel des Dienstleistungsanbieters entspricht.

Übertragen auf die vorliegende Studie wird davon ausgegangen, dass der Effekt der Prämienaufteilung auf die Erfolgswahrscheinlichkeit der Weiterempfehlung durch die von dem Empfehlungsempfänger empfundene Fairness der Prämienaufteilung verstärkt wird. Es wird erwartet, dass sich mit positiv steigender Fairnesswahrnehmung in Bezug auf die Aufteilung der Weiterempfehlungsprämie ebenso die Wahrscheinlichkeit der Annahme der Weiterempfehlung erhöht. Diese Annahme liegt der folgenden Hypothese $\mathrm{H}_{3}$ zugrunde:

$\mathbf{H}_{3}$ Der Einfluss der Prämienaufteilung auf die Erfolgswahrscheinlichkeit der Weiterempfehlung wird durch die wahrgenommene Fairness der Prämienaufteilung mediiert.

Einen Überblick der erwarteten Wirkungszusammenhänge $\left(\mathrm{H}_{1}, \mathrm{H}_{2}\right.$ und $\left.\mathrm{H}_{3}\right)$ gibt Abb. 1. 


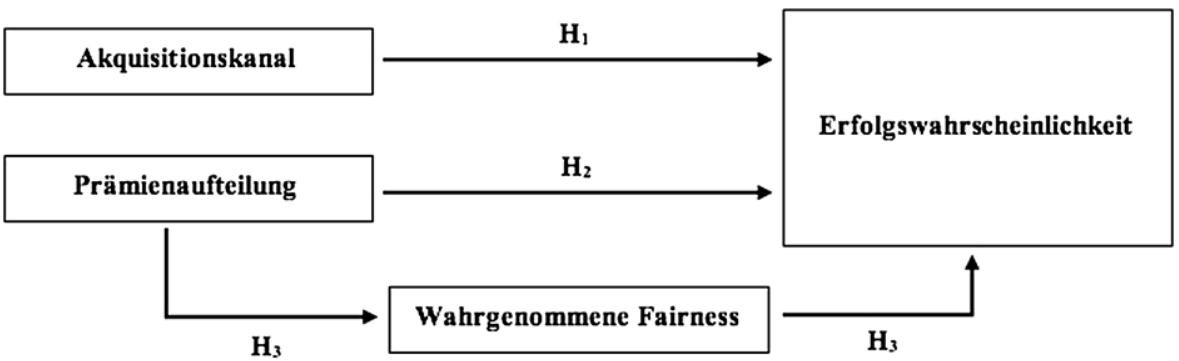

Abb. 1 Determinanten der Erfolgswahrscheinlichkeit einer Weiterempfehlung

\section{Empirische Studie}

\subsection{Methodik}

Die Überprüfung der Hypothesen $\mathrm{H}_{1}, \mathrm{H}_{2}$ und $\mathrm{H}_{3}$ erfolgte im Rahmen einer webbasierten, experimentellen Studie mittels Szenario-Technik. Variiert wurden der Akquisitionskanal des Empfehlungsgebers sowie die Prämienaufteilung zwischen Empfehlungsgeber und -empfänger. Zu Beginn der Befragung wurden die Probanden gebeten, ein Ausgangsszenario in Form einer fiktiven E-Mail zu lesen, deren Inhalt eine automatisch generierte Weiterempfehlung über das Weiterempfehlungsprogramm eines Fitness-Studios umfasste. Die Teilnehmer sollten sich vorstellen, dass sie diese Produktempfehlung von einer Person aus ihrem persönlichen Umfeld erhalten haben. Bezüglich des Empfehlungsgebers unterschied die Studie zwischen Neukunden, die selbst über das Weiterempfehlungsprogramm Kunde des Unternehmens wurden (d.h. Weiterempfehlung) und Neukunden, die über einen traditionellen Vertriebskanal akquiriert wurden (d.h. Direktmarketing). Zudem wurde innerhalb des Szenarios darauf verwiesen, dass im Falle eines erfolgreichen Vertragsabschlusses auf Basis der Weiterempfehlung eine Prämie in Höhe von $100 €$ an den Empfehlungsgeber bzw. jeweils $50 €$ an den Empfehlungsgeber sowie -empfänger ausgezahlt werden. Der vorliegenden Studie liegt ein 2 (Akquisitionskanal des Empfehlungsgebers: Weiterempfehlung vs. Direktmarketing) $\times 2$ (Prämienaufteilung: Empfehlungsgeber vs. Teilen) between-subjects Design zugrunde. Die Probanden wurden mittels softwarebasiertem Zufallstrigger annähernd auf die vier Treatments gleichverteilt.

Im Anschluss an das Ausgangsszenario sollten die Probanden ihre Intention in Bezug auf die Annahme der Weiterempfehlung angeben. Die Erfolgswahrscheinlichkeit der Weiterempfehlung als abhängige Variable wurde anhand der Fragestellung „Wie wahrscheinlich wäre es, dass Sie die Weiterempfehlung auf Basis der vorliegenden Informationen annehmen?“ über eine 7-stufige Likert Skala erhoben ( $1=\mathrm{sehr}$ unwahrscheinlich bis $7=$ sehr wahrscheinlich). Zusätzlich erfolgte die Messung der wahrgenommenen Fairness der Prämienaufteilung in Anlehnung an die Ratingskalen zur Preisfairness von Bolton, Keh und Alba (2010) anhand dreier Items (jeweils 7-stufige Likert Skalierung) ${ }^{1}$.

1 „Ich empfinde die Aufteilung der Prämie (zwischen mir und dem Sender der Empfehlung) als ...": $1=$ sehr unfair bis $7=$ sehr fair/ $1=$ nicht gerechtfertigt bis $7=$ gerechtfertigt $/ 1=$ unangemessen bis $7=$ angemessen. 
In Anlehnung an Heidenreich und Talke (2012) wurden dem Fragebogen zudem zwei Skalen zur Realitätsnähe und eine Skala zur Verständlichkeit des Szenarios hinzugefügt, um eine erfolgreiche Manipulation der beiden unabhängigen Variablen (Akquisitionskanal des Empfehlungsgebers und Prämienaufteilung) sicherzustellen. ${ }^{2}$ Es wird angenommen, dass die Manipulation bestmöglich erreicht wurde, wenn Probanden auf den 7er-Likert Skalen ( $1=$ stimme überhaupt nicht zu bis $7=$ stimme voll und ganz zu) für die drei Items jeweils mindestens einen Wert von vier angegeben haben. Befragte, die unterhalb dieses Grenzwertes lagen, wurden aus dem Sample entfernt.

\subsection{Ergebnisse}

\subsubsection{Die Erfolgswahrscheinlichkeit einer Weiterempfehlung}

An der experimentellen Studie haben 272 Personen (83,8\% zwischen 18 und 29 Jahren, Frauen: 62,0\%, Männer: 37,6\%, Divers: 0,4\%) teilgenommen. Die Ergebnisse zeigen, dass die Erfolgswahrscheinlichkeit einer Weiterempfehlung zwischen den Gruppen variiert (siehe Tab. 1). Einerseits generieren Kunden, die selbst über das Weiterempfehlungsprogramm eines Unternehmens akquiriert wurden, im Vergleich zu Kunden, die über eine Direktmarketing-Kampagne akquiriert wurden, eine signifikant höhere Erfolgswahrscheinlichkeit $\left(\mathrm{MW}_{\text {Weiterempfehlung }}=3,01\right.$, $\left.\mathrm{MW}_{\text {Direktmarketing }}=2,59, \mathrm{t}(261,403)=2,14, p<0,05\right)$. Weiterhin besteht ein höchst signifikanter Unterschied hinsichtlich der Erfolgswahrscheinlichkeit in Bezug auf die Prämienaufteilung, wobei das Teilen der Prämie zu einer signifikant höheren Erfolgswahrscheinlichkeit führt als das alleinige Entlohnen des Empfehlungsgebers $\left(\mathrm{MW}_{\text {Empfehlungsgeber }}=2,45, \mathrm{MW}_{\text {Teilen }}=3,10, \mathrm{t}(270)=-3,39, p<0,01\right)$.

Die Ergebnisse der univariaten, zweifaktoriellen Varianzanalyse verdeutlichen den signifikanten Einfluss beider unabhängiger Variablen auf die Erfolgswahrscheinlichkeit einer Weiterempfehlung (siehe Abb. 2). Es zeigt sich, dass der Akquisitionskanal des Empfehlungsgebers $(\mathrm{F}(1,268)=4,62 ; p=0,032)$ die Erfolgswahrscheinlichkeit einer Weiterempfehlung signifikant beeinflusst. Zudem besteht ein höchst signifikanter Einfluss der Prämienaufteilung zwischen dem Empfehlungsgeber und -empfänger $(\mathrm{F}(1,268)=11,01 ; p=0,001)$ auf die Erfolgswahrscheinlichkeit. Es

Tab. 1 Mittelwerte (Standardabweichung) der „Erfolgswahrscheinlichkeit einer Weiterempfehlung“

\begin{tabular}{lll}
\hline Prämienaufteilung & \multicolumn{2}{l}{ Akquisitionskanal des Empfehlungsgebers } \\
& Weiterempfehlung & Direktmarketing \\
\hline Empfehlungsgeber & $2,79(1,71)$ & $2,13(1,29)$ \\
& $(n=61)$ & $(n=67)$ \\
Teilen & $3,19(1,75)$ & $3,01(1,55)$ \\
& $(n=73)$ & $(n=71)$ \\
\hline
\end{tabular}

\footnotetext{
2 Realitätsnähe: „Das zuvor beschriebene Szenario ist realistisch.“ und „Ich konnte mich gut in die beschriebene Situation hineinversetzen.“; Verständlichkeit: „Die beschriebene Situation war leicht verständlich.".
} 
Abb. 2 Der Einfluss der „Prämienaufteilung“ und des ,Akquisitionskanals des Empfehlungsgebers“ auf die „Erfolgswahrscheinlichkeit einer Weiterempfehlung“"

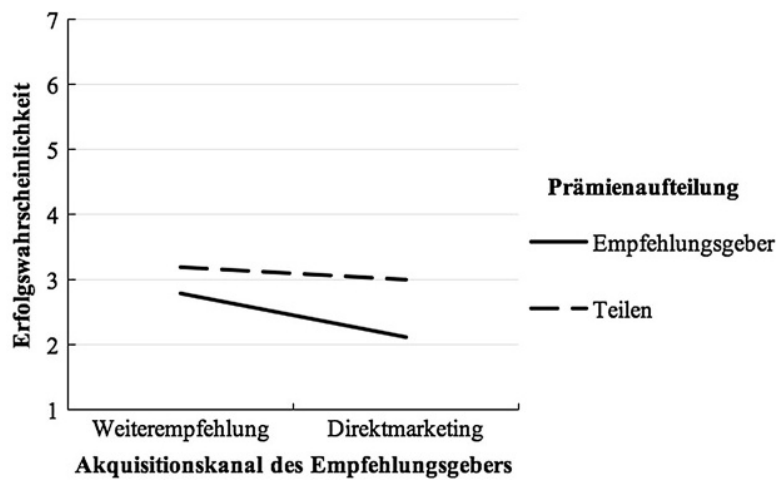

kann kein signifikanter Interaktionseffekt der beiden unabhängigen Variablen auf die abhängige Variable festgestellt werden $(\mathrm{F}(1,268)=1,51 ; p=0,220)$.

Im Rahmen eines Post-Hoc-Tests mit Bonferroni-Korrektur wurde weiterführend untersucht, welche Gruppenmittelwerte der beiden unabhängigen Variablen sich paarweise unterscheiden (siehe Tab. 1). Nicht alle Mittelwertvergleiche der Faktorstufen führten zu signifikanten Ergebnissen. Ein signifikanter Mittelwertunterschied besteht jedoch hinsichtlich des Treatments „Weiterempfehlung + Teilen“ mit dem Treatment „Direktmarketing + Empfehlungsgeber“ (Mittlere Differenz: 1,06, $p<0,05)$. Dies bedeutet, dass die Erfolgswahrscheinlichkeit der Weiterempfehlung für diejenigen Personen höher ist, deren Empfehlungsgeber selbst über das Weiterempfehlungsprogramm eines Unternehmens akquiriert wurde und zudem die Prämie geteilt wird, im Vergleich zu Personen, deren Empfehlungsgeber über Direktmarketing akquiriert wurde und die Prämie alleinig dem Empfehlungsgeber zugesprochen wird. Die Mittelwertdifferenzen zeigen zudem, dass der Unterschied in der Erfolgswahrscheinlichkeit bei diesen Faktorstufen am größten ist. Des Weiteren besteht ein signifikanter Unterschied zwischen den Treatments „Direktmarketing + Teilen“ sowie „Direktmarketing + Empfehlungsgeber“ (Mittlere Differenz: 0,88, $p<0,05$ ). Vergleicht man die Erfolgswahrscheinlichkeiten für den Fall, dass der Empfehlungsgeber über eine Direktmarketing-Kampagne akquiriert wurde, so ist die Wahrscheinlichkeit der Annahme der Empfehlung höher, wenn die Prämie zwischen dem Empfehlungsgeber und -empfänger geteilt anstatt dem Empfehlungsgeber ausgezahlt wird. Die Hypothesen $\mathrm{H}_{1}$ und $\mathrm{H}_{2}$ können somit bestätigt werden.

\subsubsection{Die wahrgenommene Fairness der Prämienaufteilung als Mediator}

Zur Überprüfung der Hypothese $\mathrm{H}_{3}$ wurde eine Mediatoranalyse durchgeführt. Verwendet wurde dabei das nicht-parametrische Bootstrapping Verfahren nach Preacher und Hayes (2004) mit $n=5000$. In einem PROCESS Model 4 (Hayes 2013) wurden die Prämienaufteilung als unabhängige Variable, die wahrgenommene Fairness als potenzieller Mediator sowie die Erfolgswahrscheinlichkeit einer Weiterempfehlung als abhängige Variable aufgenommen (siehe Abb. 3). Die Analyse zeigt, dass der Einfluss der Prämienaufteilung auf die Erfolgswahrscheinlichkeit durch die von dem Empfehlungsempfänger wahrgenommene Fairness mediiert wird 
Abb. 3 ,Wahrgenommene Fairness" als Mediator des Effekts der „Prämienaufteilung“ auf die „Erfolgswahrscheinlichkeit einer Weiterempfehlung“

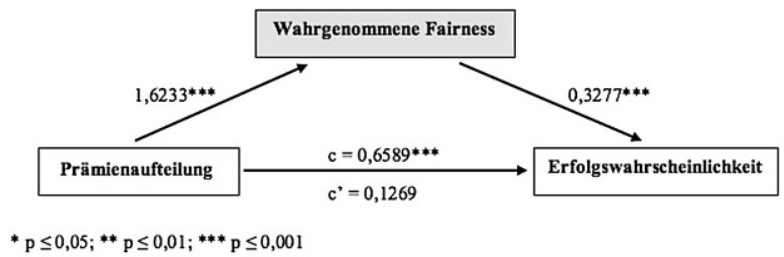

$(\mathrm{a} \times \mathrm{b}=0,5320,95 \%$-KI $[0,3112 ; 0,7877])$. Je positiver die Prämienaufteilung demnach hinsichtlich der Fairness wahrgenommen wird, desto höher ist die Erfolgswahrscheinlichkeit der Weiterempfehlung. Es besteht eine vollständige Mediation, d.h. es existiert ein mediierter Effekt zwischen der unabhängigen und der abhängigen Variable $(\mathrm{a} \times \mathrm{b})$, jedoch kein direkter Effekt (c') (Zhao et al. 2010). Zusätzlich ist der totale Effekt der Prämienaufteilung auf die Erfolgswahrscheinlichkeit höchst signifikant $(c=0,6589,95 \%$-KI $[0,2762 ; 1,0415])$. Insgesamt kann somit auch Hypothese $\mathrm{H}_{3}$ bestätigt werden.

\section{Diskussion}

Die vorliegende Studie hat durch eine differenzierte Betrachtung des Empfehlungsgebers und eine Fokussierung auf die Annahme einer Empfehlung auf Seiten des Empfehlungsempfängers wichtige Determinanten der Erfolgswahrscheinlichkeit einer Weiterempfehlung identifiziert. Die Annahme einer Weiterempfehlung wird signifikant von dem Akquisitionskanal des Empfehlungsgebers sowie der Prämienaufteilung und deren wahrgenommener Fairness beeinflusst.

\subsection{Implikationen für Forschung und Praxis}

Die Ergebnisse dieser Studie ergänzen von Wangenheim und Bayón (2004) um einen weiteren positiven Aspekt derjenigen Kunden, die über Weiterempfehlungsprogramme akquiriert wurden. Diese Kunden zeichnen sich nicht nur durch eine vergleichsweise höhere Zufriedenheit, Loyalität sowie Anzahl an positiven Weiterempfehlungen aus (von Wangenheim und Bayón 2004), sie generieren zudem eine höhere Wahrscheinlichkeit der Annahme der Weiterempfehlung durch den Empfehlungsempfänger. Auf das Weiterempfehlungsprogramm eines Versicherungsunternehmens sollten daher insbesondere solche Bestandskunden aufmerksam gemacht werden, die ursprünglich selbst über das unternehmenseigene Weiterempfehlungsprogramm akquiriert wurden. Im Anschluss an den Anbieterwechsel über das Programm, sollten Neukunden daran erinnert werden, dass sie ihre Zufriedenheit bezüglich der Entscheidung des Anbieterwechsels mit ihren sozialen Kontakten teilen können und auch die Personen aus dem Umfeld des Neukunden von den Vorteilen des Versicherers profitieren können, wenn sie über das Weiterempfehlungsprogramm den Anbieter wechseln. Zu diesem Zeitpunkt kennt der potenzielle Empfehlungsgeber einerseits die Argumente für den Anbieter sowie gegen andere Unternehmen noch sehr gut, andererseits weist er ein erhöhtes situatives Involvement auf und 
kennt den Teilnahmeprozess des Weiterempfehlungsprogramms (Richins und Bloch 1986; von Wangenheim und Bayón 2007).

Über das Weiterempfehlungsprogramm akquirierte Bestandskunden, die bereits länger bei dem Versicherungsunternehmen verweilen, sollten an die Möglichkeit der Weiterempfehlung erinnert werden. Wichtige Zeitpunkte, um den potenziellen Empfehlungsgeber auf das Weiterempfehlungsprogramm aufmerksam zu machen, sind unter anderem Situationen des Cross- bzw. Up-Selling und ein positiv verlaufener Kontakt mit dem Kundenservice des Anbieters. Entscheidet sich der Empfehlungsgeber für eine Weiterempfehlung, so sollte diese einfach, unkompliziert und schnell durchzuführen sein.

Der Prämienteilung wird in der Literatur zumeist die höchste Wahrscheinlichkeit der Abgabe einer Weiterempfehlung durch den Empfehlungsgeber zugesprochen (Jin und Huang 2014; Ryu und Feick 2007). Weitere Studien haben zudem für das Teilen einer Prämie, im Vergleich zu keiner Prämienauszahlung und einer Prämie nur für den Empfehlungsgeber, die höchste Wahrscheinlichkeit der Annahme einer Weiterempfehlung festgestellt (Sciandra 2019; Verlegh et al. 2013). Die vorliegenden Studienergebnisse sind hinsichtlich der Prämienaufteilung im Einklang mit den betrachteten Studien. Einem Versicherungsunternehmen kann bezüglich der Implementierung eines Weiterempfehlungsprogramms demnach zu einer Teilung der Prämie zwischen dem Empfehlungsgeber und -empfänger geraten werden. Zum einen erhöht eine gesteigerte wahrgenommene Fairness der Prämienaufteilung die Wahrscheinlichkeit, dass der Empfehlungsempfänger die Weiterempfehlung annimmt. Die Ergebnisse bestätigen damit die Annahme von Hong et al. (2017), dass eine gleichmäßige Teilung der Prämie als besonders fair angesehen wird. Andererseits reduziert eine faire Teilung die sozialen Kosten der Empfehlung seitens des Empfehlungsgebers und dessen Sorge um einen möglichen negativen Gesamteindruck, der bei dessen alleiniger Belohnung entstehen könnte (Jin und Huang 2014). Auf Seiten des Empfehlungsempfängers wird die Unsicherheit hinsichtlich des Motivs der Weiterempfehlung gemildert, da eine Unterstellung rein finanzieller Motive durch das Teilen der Prämie weniger auftritt und sich die Glaubwürdigkeit des Empfehlungsgebers dadurch erhöht (Kornish und Li 2010; Sniezek und Van Swol 2001).

Entscheidet sich ein Unternehmen entgegen der wissenschaftlichen Erkenntnisse dennoch, beispielsweise aus Gründen der Finanzierbarkeit ${ }^{3}$, dazu, die Prämie lediglich dem Empfehlungsgeber auszuzahlen, so wäre es ratsam, im Rahmen einer Marketingkampagne für das Weiterempfehlungsprogramm die nicht-monetären Vorteile einer Weiterempfehlung vordergründig zu bewerben. Es sollte deutlich werden, dass der Wert der Empfehlung über die Prämie hinausgeht. Einerseits erhält der Empfehlungsempfänger durch den Anbieterwechsel ein Produkt oder eine Dienstleistung, die zu seinen Bedürfnissen passt, da der Empfehlungsgeber aufgrund der bestehenden sozialen Beziehung zum Empfehlungsempfänger dessen Bedürfnisstruktur

\footnotetext{
3 In dieser Studie wurde die Prämienhöhe von $100 €$ geteilt oder vollständig an den Empfehlungsgeber ausgezahlt, wobei die finanzielle Belastung des Unternehmens gleichblieb. Unternehmen, die sich entscheiden müssen, entweder $25 €$ an den Empfehlungsgeber oder jeweils $25 €$ (50€ gesamt) an beide teilnehmenden Personen auszuzahlen, könnten dazu tendieren aufgrund der Kosteneffizienz lediglich den Empfehlungsgeber zu belohnen.
} 
kennt und dieses Wissen in die Weiterempfehlung einfließen lässt (Garnefeld et al. 2013; Schmitt et al. 2011). Wichtig ist zudem, dass die Weiterempfehlung, unabhängig von der Beziehungsstärke, als Freundschaftsdienst gesehen wird. Das heißt, dass der Empfehlungsgeber nicht die Prämie, sondern das Wohl des Gegenübers im Sinn hat (Wirtz et al. 2013). Erkennt der Empfehlungsempfänger die Reziprozität der Teilnahme an dem Weiterempfehlungsprogramm, so besteht die Möglichkeit, dass der Empfehlungsempfänger die alleinige Belohnung des Empfehlungsgebers aufgrund des Verständnisses von Geben und Nehmen weniger negativ bis zu vollkommen gerechtfertigt und positiv empfindet.

\subsection{Limitationen und zukünftiger Forschungsbedarf}

Trotz ihres Beitrags zur bisherigen Forschung im Bereich der Weiterempfehlungsprogramme, dürfen die Grenzen dieser Studie nicht außer Acht gelassen werden. Grundsätzlich enthält diese Untersuchung einen sehr großen Anteil an jungen Probanden (83,8\% zwischen 18 und 29 Jahren). Somit liegt eine gewisse Einschränkung der Repräsentativität vor, welche jedoch im Rahmen weiterer Studien für eine Zielgruppe zwischen 30 und 60 Jahren verbessert werden kann. Obwohl eine Variation des Akquisitionskanals des Empfehlungsgebers vorgenommen wurde, ist im Rahmen des $2 \times 2$-between-subjects Designs der Empfehlungsgeber lediglich als Neukunde vorgestellt worden, der selbst vor kurzem den Anbieter gewechselt hat. Ein Bestandskunde, der seit vielen Jahren bei einem Unternehmen ist, unterscheidet sich unter anderem jedoch in seinen Erfahrungen mit dem Unternehmen und seiner Loyalität von einem Neukunden (Van den Bulte et al. 2018; von Wangenheim und Bayón 2004). Es wäre daher sinnvoll, in einer Folgestudie sowohl den Einfluss von Neukunden als auch Bestandskunden als Empfehlungsgeber zu untersuchen und weiterhin jeweils deren Akquisitionskanäle zu variieren.

Bezüglich der Prämienaufteilung wurde den Probanden vorgegeben, wem und in welcher Höhe die Weiterempfehlungsprämie ausgezahlt wird. Dieses Vorgehen hat eine starke Realitätsnähe, da das Anreizsystem der Weiterempfehlungsprogramme zumeist von Unternehmen festgesetzt wird. In der Praxis existieren hingegen bereits vereinzelt Programme, die dem Empfehlungsgeber die Entscheidung über die Aufteilung der Prämie überlassen. Zukünftige Studien könnten psychologische Effekte dieser Entscheidungsübertragung untersuchen. Interessant wären zum einen die Motive für die jeweilige Prämienaufteilung und zum anderen die potenziellen Gefühle und Sorgen des Empfehlungsgebers bei dieser Entscheidung. Zudem könnten die Reaktionen des Empfehlungsempfängers auf die Offenlegung der von dem Empfehlungsgeber entschiedenen Prämienaufteilung (vor allem, wenn dieser sich die Prämie selbst auszahlt) für verschiedene Beziehungsstärken, Produkttypen aber auch Prämienarten untersucht werden.

Diese Studie betrachtet die Abgabe einer Weiterempfehlung losgelöst von dem möglichen bisherigen Empfehlungsverhalten des Empfehlungsgebers. Fraglich ist, wie eine Weiterempfehlung auf deren Empfänger und damit auf die Erfolgswahrscheinlichkeit wirkt, wenn dem Empfehlungsempfänger bekannt wird, dass der Empfehlungsgeber bereits eine erhöhte Anzahl an Empfehlungen über das Weiterempfehlungsprogramm ausgesprochen hat. Ein positiver Effekt könnte über die 
gesteigerte Erfahrung des Bestandskunden mit dem Weiterempfehlungsprogramm sowie dem Unternehmen, aber auch durch dessen ausgeprägte Loyalität begründet werden (Viswanathan et al. 2018). Einer potenziell negativen Reaktion des Empfehlungsempfängers könnte wiederum die Anwesenheit einer Prämie und der damit verbundene Vorwurf einer starken monetären Motivation zugrunde liegen (Ryu und Feick 2007; Verlegh et al. 2013).

Funding Funding Open Access funding enabled and organized by Projekt DEAL.

\section{Literatur}

Allsop, D.T., Bassett, B.R., Hoskins, J.A.: Word-of-mouth research: principles and applications. J. Advert. Res. 47(4), 398-411 (2007)

Armelini, G., Barrot, C., Becker, J.U.: Referral programs, customer value, and the relevance of dyadic characteristics. Int. J. Res. Mark. 32(4), 449-452 (2015)

Barrot, C., Becker, J.U., Meyners, J.: Impact of service pricing on referral behaviour. Eur. J. Mark. 47(7), 1052-1066 (2013)

Biyalogorsky, E., Gerstner, E., Libai, B.: Customer referral management: optimal reward programs. Mark. Sci. 20(1), 82-95 (2001)

Bolton, L.E., Keh, H.T., Alba, J.W.: How do price fairness perceptions differ across culture? J. Mark. Res. 47(3), 564-576 (2010)

Brown, J., Reingen, P.H.: Social ties and word-of-mouth referral behaviour. J. Consum. Res. 14(3), 350-362 (1987)

Dichter, E.: How word-of-mouth advertising works. Harv. Bus. Rev. 44(6), 147-166 (1966)

Diller, H.: Preispolitik. Kohlhammer, Stuttgart (2002)

Diller, H.: Preispolitik. Kohlhammer, Stuttgart (2008)

East, R., Lomax, W., Narain, R.: Customer tenure, recommendation and switching. J. Consum. Satis. Dissatis. Complain. Behav. 14(1), 46-54 (2001)

Garnefeld, I., Helm, S., Eggert, A.: Walk your talk: An experimental investigation of the relationship between word of mouth and communicators' loyalty. J. Serv. Res. 14(1), 93-107 (2011)

Garnefeld, I., Eggert, A., Helm, S.V., Tax, S.S.: Growing existing customers' revenue streams through customer referral programs. J. Mark. 77(4), 17-32 (2013)

Godes, D., Mayzlin, D.: Firm-created word-of-mouth communication: evidence from a field test. Mark. Sci. 28(4), 721-739 (2009)

Hayes, A.F.: Introduction to mediation, moderation, and conditional process analysis: A regression-based approach. Guilford, New York (2013)

Heidenreich, S., Talke, K.: Tarifwahl-Anomalien bei optionalen Mobilfunktarifen - Eine Analyse der Ursachen von Flatrate-Präferenz und Flatrate-Bias. Schmalenbachs Z. Betriebswirtsch. Forsch. 64(3), 280-307 (2012)

Heyman, J., Ariely, D.: Effort for payment: a tale of two markets. Psychol. Sci. 15(11), 787-793 (2004)

Homburg, C.: Marketingmanagement: Strategie - Instrumente - Umsetzung - Unternehmensführung. Springer Gabler, Wiesbaden (2017)

Hong, Y., Pavlou, P.A., Shi, N., Wang, K.: On the role of fairness and social distance in designing effective social referral systems. MIS Q. 41(3), 787-809 (2017)

Jin, L., Huang, Y.: When giving money does not work: The differential effects of monetary versus in-kind rewards in referral reward programs. Int. J. Res. Mark. 31(1), 107-116 (2014)

Keller, E.: Unleashing the power of word of mouth. J. Advert. Res. 47(4), 448-452 (2007)

Koch, T.: Nie war die Botschaft so wertlos wie heute (2018). https://www.wiwo.de/unternehmen/ dienstleister/werbesprech-nie-war-die-botschaft-so-wertlos-wie-heute/23163046.html, Zugegriffen: 27. Aug. 2019

Kornish, L.J., Li, Q.: Optimal referral bonuses with asymmetric information: Firm-offered and interpersonal incentives. Mark. Sci. 29(1), 108-121 (2010)

Laroche, M., McDougall, G.H.G., Bergeron, J., Yang, Z.: Exploring how intangibility affects perceived risk. J. Serv. Res. 6(4), 373-389 (2004)

Maxwell, S., Nye, P., Maxwell, N.: Less pain, same gain: The effects of priming fairness in price negotiations. Psychol. Mark. 16(7), 545-562 (1999) 
Murray, K.B.: A test of services marketing theory: consumer information acquisition activities. J. Mark. 55(1), 10-25 (1991)

Nitzan, I., Libai, B.: Social effects on customer retention. J. Mark. 75(6), 24-38 (2011)

Preacher, K.J., Hayes, A.F.: SPSS and SAS procedures for estimating indirect effects in simple mediation models. Behav. Res. Meth. Instrum. Comput. 36(4), 717-731 (2004)

Richins, M.L., Bloch, P.H.: After the new wears off: The temporal context of product involvement. J. Consum. Res. 13(2), 280-285 (1986)

Ryu, G., Feick, L.: A penny for your thoughts: referral reward programs and referral likelihood. J. Mark. 71(1), 84-94 (2007)

Schmitt, P., Skiera, B., Van den Bulte, C.: Referral programs and customer value. J. Mark. 75(1), 46-59 (2011)

Sciandra, M.R.: Money talks, but will consumers listen? Referral reward programs and the likelihood of recommendation acceptance. J. Mark. Theor. Pract. 27(1), 67-82 (2019)

Sniezek, J.A., Van Swol, L.M.: Trust, confidence, and expertise in a judge-advisor system. Organ. Behav. Hum. Dec. 84(2), 288-307 (2001)

Van den Bulte, C., Bayer, E., Skiera, B., Schmitt, P.: How customer referral programs turn social capital into economic capital. J. Mark. Res. 55(1), 132-146 (2018)

Verlegh, P.W.J., Ryu, G., Tuk, M.A., Feick, L.: Receiver responses to rewarded referrals: the motive inferences framework. J. Acad. Mark. Sci. 41(6), 669-682 (2013)

Villanueva, J., Yoo, S., Hanssens, D.M.: The impact of marketing-induced versus word-of-mouth customer acquisition on customer equity growth. J. Mark. Res. 45(1), 48-59 (2008)

Viswanathan, V., Tillmanns, S., Krafft, M., Asselmann, D.: Understanding the quality-quantity conundrum of customer referral programs: effects of contribution margin, extraversion, and opinion leadership. J. Acad. Mark. Sci. 46(6), 1108-1132 (2018)

Von Wangenheim, F., Bayón, T.: Satisfaction, loyalty and word of mouth within the customer base of a utility provider: differences between stayers, switchers and referral switchers. J. Consum. Behav. 3(3), 211-220 (2004)

Von Wangenheim, F., Bayón, T.: The chain from customer satisfaction via word-of-mouth referrals to new customer acquisition. J. Acad. Mark. Sci. 35(2), 233-249 (2007)

Wirtz, J., Chew, P.: The effects of incentives, deal proneness, satisfaction and tie strength on word-ofmouth behaviour. Int. J. Serv. Ind. Manag. 13(2), 141-162 (2002)

Wirtz, J., Orsingher, C., Chew, P., Tambyah, S.K.: The role of metaperception on the effectiveness of referral reward programs. J. Serv. Res. 16(1), 82-98 (2013)

Xia, L., Monroe, K.B., Cox, J.L.: The price is unfair! A conceptual framework of price fairness perceptions. J. Mark. 68(4), 1-15 (2004)

Xiao, P., Tang, C.S., Wirtz, J.: Optimizing referral reward programs under impression management considerations. Eur. J. Oper. Res. 215(3), 730-739 (2011)

Zeithaml, V.A.: How consumer evaluation processes differ between goods and services. In: Donnelly, J.H., George, W.R. (Hrsg.) Marketing of services, S. 186-190. American Marketing Association, Chicago (1981)

Zhao, X., Lynch, J.G., Chen, Q.: Reconsidering Baron and Kenny: Myths and truths about mediation analysis. J. Consum. Res. 37(2), 197-206 (2010)

Lisa-Marie Klopfer M.Sc., ist wissenschaftliche Mitarbeiterin des Lehrstuhls für BWL, insb. Versicherungsmarketing, der Friedrich-Alexander-Universität Erlangen-Nürnberg

Martina Steul-Fischer Prof. Dr., ist Inhaberin des Lehrstuhls für BWL, insb. Versicherungsmarketing, der Friedrich-Alexander-Universität Erlangen-Nürnberg 